\title{
Transmission Power Adaption Scheme for Improving IoV Awareness Exploiting Evaluation Weighted Matrix based on Piggybacked Information
}

\author{
Ali Safa Sadiq ${ }^{1}$, Suleman Khan ${ }^{1}$, Kayhan Zrar Ghafoor ${ }^{2,3}$, Mohsen Guizani ${ }^{4}$, Seyeali Mirjalili ${ }^{5}$ \\ ${ }^{1}$ School of Information Technology, Monash University, 47500 Bandar Sunway, Malaysia \\ ${ }^{2}$ Department of Computer Science and Engineering, Shanghai Jiao Tong University, Shangahai, China \\ ${ }^{3}$ Department of Computer Science, Faculty of Science, Cihan University, Erbil, KRG, IRAQ \\ ${ }^{4}$ Department of Electrical and Computer Engineering, University of Idaho, USA \\ ${ }^{5}$ Institute for Integrated and Intelligent Systems, Griffith University, Brisbane, Australia
}

\begin{abstract}
As part of the new era Internet of Things, an evolved form of Vehicle Ad-hoc Networks has recently emerged as the Internet of Vehicles (IoV). IoV has obtained a lot of attention among smart vehicle manufactures and illustrations due to its promising potential, but there are still some problems and challenges that need to be addressed. Transmission error occurs when an emergency message is disseminated to provide traffic awareness, and vehicles have to increase their channel transmission power to ensure further coverage and mitigate possible accidents. This might cause channel congestion and unnecessary power consumption due to an inaccurate transmission power setup. A promising solution could be achieved via periodically and predictively evaluating channel and GEO information that is transmitted over piggybacked beacons. Thus, in this paper we propose a Transmission Power Adaptation (TPA) scheme for obtaining better power tuning, which senses and examines the probability of channel congestion. Afterwards, it proactively predicts upcoming channel statuses using developed evaluation weighted matrixes, which observes correlations between coefficients in the variance of estimated metrics. Considering beacon transmission error rate, crowding inter-vehicle distance, and channel delay, the matrix is periodically constructed and weighted so that each metric is based on a predefined threshold value. Eventually, predicted channel status is used as an indicator to adjust transmission power. This leads to decreased channel congestion and better awareness in IoV. The performance of the proposed TPA scheme was evaluated using OMNeT++ simulation tools. The simulation results show that our proposed TPA scheme performs better than existing method in terms of overall throughput, average beacon congestion rate, beacon recipient rate probabilities, channel-busyness, transmission power over distance, and accident probabilities.
\end{abstract}

Index Terms - Energy Efficient Devices, Energy Harvesting, Crowd Sensing and Crowd Sourcing, Efficient Communications and Networking, Machine-to-Machine Communications, Internet of Vehicles

\subsection{INTRODUCTION}

Internet of Vehicles (IoV) has emerged as a promising technology that has received significant attention in the field of wireless communications in the last decade. This type of communication uses a wireless communication systems known as Vehicular Ad-hoc Networks (VANETs). Increasing numbers of vehicles on roads has made communications more challenging, requiring dynamic updates of recent network status. The process of locally and periodically broadcasting information that indicates the network status is part of the communication patterns in VANETs. In order to obtain information related to other communicating partners, the vehicle should receive beacon messages that are periodically broadcasted via neighborhood vehicles. Beacon message contains a vehicle's identifier, geographical position, velocity, utilized transmission power, and other information related to channel characteristics [1], [2], [3]. The information obtained from these beacons will be utilized later on by vehicular applications to improve performance.

Many applications has been introduced by both research and industrial societies to obtain solutions to daily traffic issues. For instance, applications have been developed to improve road traffic safety, finding the shortest path for drivers, and many other entertainment applications. These applications require continues monitoring of network status to improve performance. Extremely dynamic vehicular topology and channel characteristics can cause a frequent transmission errors with the running of IoV road safety applications. Therefore, guaranteeing an efficient beaconing mechanism that can provide continues information exchanges in highly dynamic VANETs environments would result in extending the lifetime of high priority messages.

Numerous studies have paid attention to introducing efficient methods to manage the channel load produced by broadcasted beacons. A number of the proposed methods deal with vehicular channel load matter by adjusting beacon periodicity or generation rate, whereas a few more studies proposed regulating transmission power to control the number of vehicles within the coverage area competing for the CCH channel. Certain advantages and limitations are obtained by using any of the proposed methods. In other words, each of the aforementioned methods is valuable in some particular scenarios and less functional in some others.

A solution that could be achieved here is the hybridization of both concepts, to create a balance between beacon generation rate adjustment and transmission power adaptation methods. However, lacking a deep understanding of what could be the negative aspects of every method, it is quite hard to meet the requirements of safety applications with an adaptive transmission power that maintains 
an efficient channel with less power consumption. In this paper, we focus on designing an efficient transmission power adaptation scheme that could ensure the vehicles' awareness of high priority messages without affecting channel load, creating possible congestion and high error rates, in particular, the possible energy harvesting that can be achieved via the use of the associated piggybacked forwarded messages when adjusting transmission power, using a developed evaluation weighted matrix that examines the correlations between the variance coefficients of estimated metrics in shared vehicle channels and GEO information [4, 5].

The remainder of this paper is structured as follows. In section 2.0, the related works that were recently proposed in the area of study will be presented besides a comparison table will be given. Section 3.0 will introduce the proposed methodology of our TPA scheme based developed evaluation weighted matrix and discussion will be given on the research design. Simulation results and performance analysis are elaborated in Section 4.0, followed by the conclusion and future work in Section 5.0.

\section{0}

\section{RELATED WORKS}

Numerous proposals have been presented in the literature for the efficient distribution of safety messages between vehicles. In order to reduce packet level interference, the authors in [6] conducted a performance analysis to distinguish possible approaches to improve VANET performance in support safety and introduced an algorithm named Distributed-Fair Transmit Power Adjustment (D-FPAV) based on Vehicular Ad hoc networks. This preserves beacon loads, allowing the active safety applications to meet a firm deadline for event-driven messages. Moreover, they proposed a dissemination technique based relay node self-election that can provide fast beacon distribution towards the destination.

In [7], [8], the authors carried out a performance comparison using simulations and created measurements based on routing metrics such as packet delivery ratio, packet delay, average path length, throughput, and routing overhead [9], [10]. They proved that existing research showed poor performance for current VANET systems. A new scheme was proposed by authors in [11] to dynamically analyze power transmission based on a numerical model. Thus, they achieved a mechanism to maintain the transmission power of periodic beacon messages. The main benefit of the introduced scheme is that the vehicle calculates the number of received or lost beacons per second from the nearest neighboring vehicles.

Afterwards, the authors in [12] proposed an adaptive power control mechanism modeled on traffic and propagation. In order to model power propagation, lognormal shadowing was used for a highway vehicular scenario. Real highway data has been used to model vehicular traffic distribution from late night to the daytime rush hour. The author in [13] proposed Fair Power Adjustment for Vehicular environments (FPAV), with a centralized power control algorithm that offered a way to adjust channel load issues in a VANET environment. This was achieved by maximizing the minimum transmission range for all vehicles in a synchronized way by analyzing piggyback beacon information received from neighboring vehicles. Accordingly, safety messages will disseminate to all neighboring vehicles as they autonomously select themselves as a subsequent forwarder. The proposed mechanism favors the farthest vehicle to be a next beacon message forwarder.

Mittag et al. proposed a framework in [14] as a way to conduct a fair comparison between single-hop transmissions at high transmission power and multi-hop transmissions relayed at a lower transmission power. Mittag et al. goal was to know whether or not an efficient multi-hop beacon could decrease channel load. He found that a single-hop should be used for a beacon system while multi-hop should be utilized to provide a higher coverage area. It is important to mention that broadcasting with maximum power generates a broadcast storm issue.

In spite of the aforementioned proposed methods, the majority only considered channel access time according to message importance and required delay, without taking into account transmission power adaption, or the prioritization of messages according to channel status, which could improve channel throughput and reduce end-to-end delay [15-18]. In fact, the main goal of transmission power adaptation in VANETs is to increase throughput, network coverage, and link lifetime [19-21]. These desired power control objectives are quite challenging in vehicular networks since network topology and communication method between vehicles has unique characteristics compared with other mobile communications. V2V communication was introduced to facilitate traffic safety applications on the road. For these reasons, reliability and real-time safety applications should be considered when designing a power control mechanism within vehicular networks. A detailed analysis should be considered to score the evaluation metrics of wireless channel characteristics and GIO information as a way to make a better judgment in adjusting transmit power for better support of IoV applications. Consequently, power adaptation essentially need to be fair crossways vehicles within their transmission ranges. Employing fair dynamic adaption involves information sharing among vehicles consisting their observations of the topology and wireless channel states [22]. To this end, these considerable challenges have encouraged us to look forward introducing a more efficient power control approach called Transmission Power Adaption scheme, which exploiting evaluation weighted matrix based on piggybacked information. Table 1 shows a summary of the drawbacks in related studies transmission power adaptation techniques. 


\section{0}

PROPOSED TPA BASED EVALUATION WEIGHTED MATRIX

As a perspective example, Figure 1 shows two examples of source vehicles and their accessible communication range and the farthest target vehicles, which are symbolized as $T x$ and $R$, correspondingly. At the first scenario, when the source vehicle disseminates a safety message to nearby vehicles, the nearby vehicles will be receiving the messages and extracting the piggybacked information. We have proposed a Crowding-Inter-Vehicle-Distance Table $\left(C_{-}\right.$Int-V_DT) that will be calculated and generated according to the ranked crowding distance concept between the sender and a group of associated receiver vehicles. The calculated C_Int-V_D by source vehicle towards its associated neighbors will build the awareness to adapt its power of transmission to obtain a $T x$ range within $R$ range.

Table 1: Summary Table of related works

\begin{tabular}{|c|c|c|c|c|c|}
\hline $\begin{array}{l}\text { Power Adaption } \\
\text { Approaches }\end{array}$ & Categorization & Objective & Summary & Simulator & Remarks on performance \\
\hline Guan et al. [20] & TPA & $\begin{array}{l}\text { Achieving consistency and } \\
\text { maintaining latency for } \\
\text { supporting lively safety } \\
\text { applications }\end{array}$ & $\begin{array}{l}\text { It uses piggybacked } \\
\text { information of Power } \\
\text { control at the beacon } \\
\text { header }\end{array}$ & $\begin{array}{l}\text { OPNET } \\
\text { simulator }\end{array}$ & $\begin{array}{l}\text { The proposed algorithm maintains } \\
\text { enhanced message loss rate in } \\
\text { comparison with non-power } \\
\text { control, but it was tested with } \\
\text { small No. of nodes. }\end{array}$ \\
\hline Artimy et al. [23] & TPA & $\begin{array}{l}\text { Maintaining high range of } \\
\text { connectivity }\end{array}$ & $\begin{array}{l}\text { It estimates the density of } \\
\text { local vehicles traffic }\end{array}$ & RoadSim & $\begin{array}{lrr}\text { Simulation } & \text { shows } & \text { dynamic } \\
\text { transmission } & \text { range } & \text { achieves } \\
\text { optimal connectivity } & \text { but } \\
\text { estimating an optimal } & \text { traffic } \\
\text { density still an open issue } & \end{array}$ \\
\hline $\begin{array}{l}\text { Torrent-Moreno et } \\
\text { al. [24] }\end{array}$ & TPA & $\begin{array}{l}\text { Decreasing beacon's load } \\
\text { and firm message } \\
\text { distribution }\end{array}$ & $\begin{array}{l}\text { Set priorities for } \\
\text { emergency messages and } \\
\text { receiver and disseminate } \\
\text { them based on local } \\
\text { election process by each } \\
\text { vehicle }\end{array}$ & $\begin{array}{l}\mathrm{ns}-2 \\
\text { simulator }\end{array}$ & $\begin{array}{l}\text { Their protocol achieves better } \\
\text { performance in allocating time for } \\
\text { accessing a channel and ensure } \\
\text { the recipient of messages. More } \\
\text { analysis is needed with more time }\end{array}$ \\
\hline Samara et al. [11] & TPA & $\begin{array}{l}\text { Improve message recipient } \\
\text { rate with the consideration } \\
\text { of channel status }\end{array}$ & $\begin{array}{l}\text { Each vehicle computes an } \\
\text { interval time of the } \\
\text { number of successfully } \\
\text { received and lost beacons }\end{array}$ & $\begin{array}{l}\text { Numerical } \\
\text { analysis }\end{array}$ & $\begin{array}{l}\text { The proposed algorithm inquires } \\
\text { more accurate analysis and } \\
\text { evaluation }\end{array}$ \\
\hline Ning. et al. [25] & $\begin{array}{l}\text { Review on } \\
\text { wireless } \\
\text { commination } \\
\text { in VANET }\end{array}$ & $\begin{array}{l}\text { Identifying the future } \\
\text { research in vehicular } \\
\text { communication }\end{array}$ & $\begin{array}{l}\text { There are still various } \\
\text { challenges in enabling } \\
\text { Internet of Vehicles (loV), } \\
\text { one of them is safety } \\
\text { message dissemination }\end{array}$ & None & $\begin{array}{l}\text { The authors highlighted that DSRC } \\
\text { MAC doesn't support efficiently } \\
\text { safety messages dissemination as } \\
\text { it works based on contention } \\
\text { since of high possibility of packet } \\
\text { collision resulted from inefficacy } \\
\text { with transmission power } \\
\text { allocation }\end{array}$ \\
\hline Mittag et al. [14] & TPA & $\begin{array}{l}\text { Reducing wireless channel } \\
\text { load }\end{array}$ & $\begin{array}{l}\text { Broadcasting using a } \\
\text { maximum in an } \\
\text { emergency cases power }\end{array}$ & None & $\begin{array}{l}\text { One dimensional and optimal } \\
\text { scenario that could lead to } \\
\text { broadcast storm and decrease the } \\
\text { network performance }\end{array}$ \\
\hline Hyung [2] & $\begin{array}{l}\text { Low power } \\
\text { routing }\end{array}$ & $\begin{array}{l}\text { Build a model for analyzing } \\
\text { the power consumption for } \\
\text { wireless channel }\end{array}$ & $\begin{array}{l}\text { Contract a mapping } \\
\text { between distance and } \\
\text { power consumption }\end{array}$ & WiSeR & $\begin{array}{l}\text { Considering distance affect } \\
\text { doesn't provide a complete view } \\
\text { on power level }\end{array}$ \\
\hline
\end{tabular}

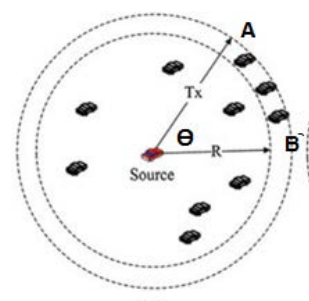

(a)

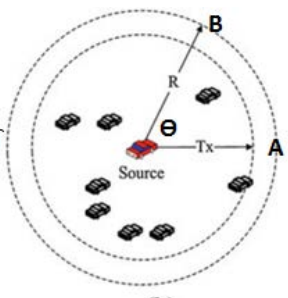

(b)

Figure 1. Effect of crowding distance of the variable target and transmission range on the power control. $\mathbf{a} T x$ is larger than $R$. $\mathbf{b} R$ is larger than $T x$ 
To lead the evaluation process of the dens of surrounding area of Source 1 and 2 vehicles in Figure 1, we suggested defining a crowded-inter-vehicle-distance-comparison operator $(<c-v-d)$. Each vehicle $i$ has two features, no dominated neighbors $B_{\text {Rank }}^{-}$and dominated neighbors $B_{\text {Rank }}$. Moreover, the crowding inter-vehicle distance to the nearby vehicles $B_{D}$. The following definition based on the abovementioned features defines the $<c-v-d$ :

$$
\widehat{\mathrm{B}} \prec c_{v_{d}} \mathrm{~B}=\widehat{\mathrm{B}}_{\text {Rank }}<\mathrm{B}_{\text {Rank }} \vee\left(\mathrm{B}_{\text {Rank }}=\mathrm{B}_{\text {Rank }} \wedge \mathrm{B}_{\mathrm{D}}>\mathrm{B}_{\mathrm{D}}\right.
$$

The $<c-v$-d gives a higher rank for those vehicles with higher $B_{\text {Rank }}$ when the ranking performed between two vehicles falls at similar rank. While considering the density level of neighboring vehicles, the preferred solution will be the one who obtained higher $\left\langle c-v-d\right.$. Base on this concept the Crowding inter-vehicle distance $\left(C_{-} I n t-V_{-} D\right)$ was calculated. Utilizing the formed transmission radius that obtained based on deployed power transmission and the degree of Theta ${ }^{\theta}$ of the formed circular communication range, the distance can be calculated as a length between point A and B in Figure 1.

$$
\begin{gathered}
\overline{A B}=\theta, \quad \frac{\theta}{360^{\circ}} 2 \pi R \\
f\left(B_{D_{i}}\right)=\sqrt{\sum_{i-1}^{n}\left(\overline{A_{i}}-\overline{B_{i}}\right)^{2}}
\end{gathered}
$$

In order to achieve vehicular information awareness, each received beacon should be processed in such way to get the information about neighbor's vehicle. In our proposed TPA scheme, beacon should piggyback all suggested information about transmission power, Time of carrying a beacon $T_{-} c$, Time of forwarding a beacon $T \_f$, and $C_{-} I n t-V_{-} D$; that are lending a hand to receiver distinguish the appropriate power for transmission that can be used, TPA_val.

\begin{tabular}{|c|c|c|c|c|c|c|c|c|}
\hline Bytes:2 & 4 & $\mathbf{6}$ & $\mathbf{6}$ & $\mathbf{6}$ & $\mathbf{2}$ & $\mathbf{2}$ & $\mathbf{0 - 2 3 0 4 +}$ & $\mathbf{4}$ \\
\hline $\begin{array}{c}\text { Frame } \\
\text { Control }\end{array}$ & Duration & $\begin{array}{c}\text { Address } \\
\mathbf{1}\end{array}$ & $\begin{array}{c}\text { Addres } \\
\mathrm{s} 2\end{array}$ & $\begin{array}{c}\text { Address } \\
\mathbf{3}\end{array}$ & $\begin{array}{c}\text { Sequence } \\
\text { Control }\end{array}$ & $\begin{array}{c}\text { QoS } \\
\text { Control }\end{array}$ & $\begin{array}{c}\text { Frame } \\
\text { body }\end{array}$ & FCS \\
\hline
\end{tabular}

Figure 2: IEEE 802.11p MAC header format. [26]

In this case, the power information added is piggybacked to the current beacon used in VANET. Basically, every beacon message has its unique sequence number which takes place from MAC layer. Based on the standard of IEEE 802.11p, the sequence control field SL is allocated in an 802.11 MAC header utilizing two bytes, which can be adapted to distinguish any possible collision and channel load in the network, as presented in Figure 2 [26].

Afterwards, each vehicle extracts the piggybacked information that received via modified beacons, presented in Figure3, and then utilizes them in calculating current channel status and distinguishes if any possible network congestion could be faced during that time. We can infer the channel behavior from beacon arrival when beacon arrives meaning that the network is not congested, whereby it could be congested when beacon fails to reach its destination or rely-vehicles. Thus, the percentage of congestion could be obtained by identifying the number of beacons unsuccessfully received by their destination. It's important mentioning that, our proposed TPA scheme not focusing on maintains the beacon generation rate. Therefore, we assumed that each vehicle generating 10 beacons per second, and then supposedly every single second as each neighbor vehicle should receive 10 beacons in average per one second out of $n$ number of neighborhood vehicles. Afterwards the variance of received beacons is calculated by considering the accumulated received beacons from neighboring vehicles and the obtained percentage, thus an absolute value will be obtained indicating the beaconing activities of $i_{t h}$ vehicle. The total number of sent beacons in the area of evolution could be calculated as in Equation $1, B_{\text {sent }}$. When $B_{n o}$ is the number of the sent beacons per vehicle $n$ which is equal to10, $E_{\text {int }}$ is the interval of evolution that is 10 seconds and $n$ is the number of vehicles in the evaluated area.

$$
B_{\text {sent }}=B_{n o} \times E_{\text {int }} \times n, B_{\text {recieved }}=\frac{\sum_{i=1}^{n} B_{\text {received }}}{\text { Total } B_{\text {sent }}}
$$




$$
\begin{aligned}
& \begin{array}{|l|l|l|l|l|l|l|l|l|l|l|l|l|l|}
\hline \text { Seq } & \text { Int } & \text { TS } & \text { ELP } & \text { Pos } & \text { Spd } & \text { Dir } & \text { Maxp } & \text { Minp } & \text { TPA_val } & T-C & T-\text { F } & \text { C_Int-V_D } & \text { P_val } \\
\hline
\end{array} \\
& \text { Variance }_{B_{\text {received }}}=\left|\frac{\sum_{i=1}^{n} B_{\text {received }}-B_{\text {Sent }}}{B_{\text {sent }}}\right|=\left\{\begin{array}{c}
- \text { Variance_B} B_{\text {received }}, \text { Variance_ } B_{\text {received }} \leq 0 \\
\text { Variance_B } B_{\text {received }}, \text { Variance_B } B_{\text {received }}>0
\end{array}\right.
\end{aligned}
$$

Equation 2 calculates the variance measure between the averages sent and received beacons every 10 second interval. For instance, based on illustrated scenario by Figure 4, when vehicle B intending to analyze the percentage of received beacons, out of the actually received from vehicle A the percentage of $B_{\text {received }}$, (you could refer to Table 2 to note all the TPA's parameters), was $40 \%$ and $60 \%$ was failed beacons, as beacon 3-7 and 10 are lost, refer to Table 3 . In order to be more precise, we have considered the $C-I n t-V_{-} D T$ of Vehicle B as the percentage of received beacons inversely proportional to crowding distance. We could obtain the distance by using the aforementioned process that illustrated by example in Figure 2 , which are all encapsulated as piggybacked information on generated beacons. In order to make it easier for analysis, we proposed a Table called $C_{-} I n t \_V \_D T$ which includes the vehicle IDs, percentage of reception, number of beacon received and distance the between source and destination, Table 4. The probability of error with received beacons could be obtained as $B_{e}$ calculated bellow:

$$
B_{e}=\frac{\left(\text { Variance } B_{\text {received }}\right)}{\frac{C_{I n t} V_{D}}{\operatorname{Max}}}
$$

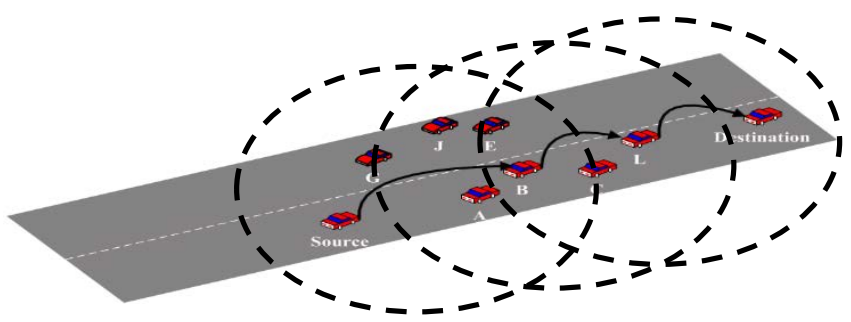

Figure 4: Vehicle B receives beacons from neighbors as a relay-node between source and destination vehicles

Table 2: Transmission Power Adaptation Scheme parameters

$\begin{array}{lr}\text { Percentage of Received Beacons } & B \text {-received } \\ \text { Beacons received per sec } & B / s \\ \text { Error computed per single vehicle } & B_{e} \\ \text { Vehicles number } & V_{-} n \\ \text { Total error for single channel with V_n } & \left.\text { Total } B_{e}\right) \\ \text { Crowd_Inter-vehicle distance } & C \_I n t-V_{-} D \\ \text { Averaged variance of total received beacons from V_n } & \text { Variance_B } B_{\text {received }} \\ \text { Channel Delay } & D_{\text {Channel }} \\ \text { Beacon carrying time } & T_{c} \\ \text { Beacon forwarding time } & T_{f} \\ \text { Normalized factor of current channel status } & C C_{-} s \\ \text { Maximum power received in the field MaxP from neighbors } & \text { MaxP } \\ \text { Minimum power received in the field MaxP from neighbors } & M i n P \\ \text { Power deference between MaxP and MinP power } & P-D e f \\ \text { Priority value } & P \_v a l \\ \text { Circuit power consumption } & P_{c}\end{array}$


Table 3: Sequence number received from five neighbouring vehicles (refer to Figure3).

\begin{tabular}{|c|c|c|c|c|c|c|c|c|c|c|}
\hline $\begin{array}{c}\text { VEHICLE } \\
\text { ID }\end{array}$ & \multicolumn{8}{|c|}{ SEQUENCE NUMBER OF BEACON } \\
\hline Source & 1 & 2 & 3 & 4 & & 6 & 7 & & 9 & 10 \\
\hline A & 11 & 12 & & & 15 & & & 18 & 19 & 20 \\
\hline B & 21 & 22 & & & & & & 28 & 29 & \\
\hline L & 31 & 32 & 33 & 34 & 35 & 36 & 37 & & 39 & \\
\hline Destination & 41 & 42 & 43 & & & & 47 & & 49 & 50 \\
\hline
\end{tabular}

Table 4: Proposed $C \_I n t-V \_D T$ example.

$$
\begin{aligned}
& \begin{array}{|c|c|}
\hline \begin{array}{c}
\text { VEHICLE } \\
\text { ID }
\end{array} & \begin{array}{c}
\text { C_Int- } \\
\text { V_D }
\end{array} \\
\hline \text { Source } & 190 \mathrm{~m} \\
\hline \text { A } & 100 \mathrm{~m} \\
\hline \text { B } & 150 \mathrm{~m} \\
\hline \text { L } & 130 \mathrm{~m} \\
\hline \text { Destination } & 270 \mathrm{~m} \\
\hline
\end{array} \\
& \mathrm{D}_{\text {Channel }}=\sum_{V \_n=1}^{V \_n}(T c+T f) \\
& \operatorname{CoV}_{C_{\text {Int }_{D}}}=\sum_{V_{n}=1}^{V_{n}}\left(\mathrm{~B}_{\mathrm{e}}-\overline{\mathrm{B}_{\mathrm{e}}}\right)\left(C_{\text {Int }_{V_{D}}}-\overline{\text { Int }_{V_{D}}}\right) /\left(V_{n}-1\right) \\
& \text { CoV }_{\text {Time_Delay }}=\sum_{V_{n}=1}^{V_{n}}\left(\mathrm{~B}_{\mathrm{e}}-\overline{\mathrm{B}_{\mathrm{e}}}\right)\left(\mathrm{D}_{\text {Channel }}-\overline{\mathrm{D}_{\text {Channel }}}\right) /\left(V_{n}-1\right)
\end{aligned}
$$

By referring to the given scenario in Figure 4, we could identify from generated $C \_I n t-V \_D T$ in Table 4 that vehicle $B$ could manage successfully to send an average 4 beacons per second. After applying Equation 1 and present it in a percentage form $B_{\text {received }}=40 \%$, from Equation 2 Variance $_{B_{\text {received }}}=0.550$, and applying Equation 3 value 1 has obtained; which indicating that 1 beacon could be failed every 1 meter of $C_{-} I n t-V_{-} D$. Thus, when the $C_{-} I n t-V_{-} D$ for vehicle $B$ grows for 1 meter another 1 beacon in average could be lost from the current neighboring vehicles and the possible percentage of received beacons in the future time $t+1$ will be reduced by roughly $25 \%$ from its existing percentage to be $15 \%$ instead of $40 \%$. This is due to the fact that when the $C_{-}$Int $-V_{-} D$ increases it has a positive correlation with Variance $_{B_{\text {received }}}$ metric, hence the ratio of dropped beacons will be negatively impacted and will contribute in degrade the current channel status $C C_{s}$. To have clear figure if the impact of calculated $C \_I n t-V \_D$ on the error ratio, Equation 5 computes the coefficient of variation for beacon error rate with respect to inter-vehicle crowding distance $\operatorname{CoV}_{C_{\operatorname{Int}_{V_{D}}}}$.

By using Equation 3 we can predict the total beacon error of a particular channel of vehicle $i$. We have also considered other important measure metric to be included in the proposed evaluation matrix, which is the accumulative time delays of wireless channel $\mathrm{D}_{\text {Channel }}$, that consists of $T_{-} c$ and $T_{-} f$, average time of carrying and forwarding a beacon during the time of evaluation of 10 seconds. Thus, the coefficient of variation is calculated using Equation 6 as a way to estimate the impact of the consumed times $T_{-} c$ and $T_{-} f$ on the channel error rate, which representsB $\mathrm{e}_{\mathrm{e}}$. CoV $V_{\text {Time }}$ Delay is calculated with respect to the average channel delay. A better estimation of channel condition can be achieved by accurately obtaining the values of $T \_c$ and $T \_f$. As a way to achieve that we have considered the probability of vehicle distribution. Therefore, we assumed that the number of vehicles transient a cretin point per time $t$ is represented by Poisson probability with a mean $(1 / \lambda)$, as was suggested in [27]. Accordingly, the vehicles are traveling as $\lambda$ vehicles per $t$. As a result, an exponential distribution with $\lambda$ factor could be applied to represent the time intervals. Moreover, we supposed that there are $N$ separate intensities of steady vehicle's velocities $V(i=1, \ldots, n)$ on the highway. Hence, by applying the separate intensities of velocity, we can obtain an arrival rate of vehicles at each intensity of velocity the same as $\lambda_{i}(i=1, \ldots, n)$, where $\_n i=1=\lambda$; consequently, the occasional probability of each velocity is $P \lambda_{i} / \lambda$. 


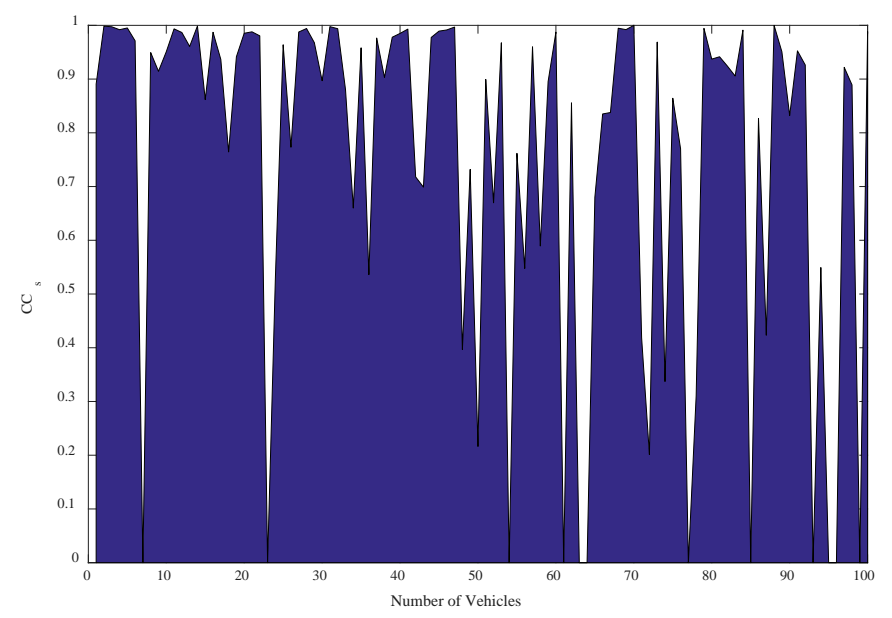

Figure 5: Plotted areas of computed Current Channel status factor $\mathrm{CC}_{\mathrm{s}}$ for 100 vehicles

Thus, each vehicle can easily analyze the piggybacked information that received from beacon which consists the information about transmission power and others as presented in Table 5. From the given example of an active beacon coming from one vehicle, relay vehicles can analyze at any instant the transmission power of received beacons from vehicle " $B$ " (as an example), the received power depends on crowding distance between vehicle " $B$ " and other neighboring vehicles. As an example, when vehicle " $B$ " transmits with power less than 20 , the beacon might not reach further distances but, it attempts less congestion that allows other vehicles to disseminate the emergency messages with less errors, in case of $19.79 \mathrm{dBm}$ equivalents to $0.095 \mathrm{Watt}$, refer to Table5. It is important to note that we have represent the power in the signal form measured by "dbm" while the numerical calculation was conducted by considering the equivalent transmission power in "Watt".

Considering both $\mathrm{CoV}_{\text {Int }_{V_{D}}}$ and $\mathrm{CoV}_{\text {Time_Delay }}$ the current channel status for each vehicle will be calculated using Equation 7. The output will be a scale value between 0 to 1 where 0 indicating the worst channel status "Min" and 1 reflecting the optimum status "Max". For having clear understanding, Figure 5 visualized using numerical analysis the plotted areas of computed $C C_{s}$ for 100 vehicles. From this figure we can observe the obtained channel status variation, considering random changes with average received beacons, $T_{-} c, T_{-} f$ and vehicles distribution reflected to the $\operatorname{CoV}_{C_{I_{n t} V_{D}}}$. Using this estimation mechanism each vehicle can adapt its transmission power based upon the channel status.

$$
C C_{S}=\left|1-\left(\frac{{ }^{C o V} C_{\text {IntV }_{D}}{ }^{\text {CoV }} \text { Time_Delay }_{-}}{2}\right)\right|
$$

Table 5: Active example of a beacon with piggybacked information

\begin{tabular}{|c|c|c|c|c|c|c|c|c|c|c|c|c|c|}
\hline Seq & Int & Ts & ELP & Pos & Spd & Dir & MaxP & MinP & TPA_val & T_c & T_f & $\begin{array}{c}\text { C_Int- } \\
\text { V_D }\end{array}$ & P_val \\
\hline 22 & 100 & 2.04 & B & $\begin{array}{c}3^{\prime} 48^{\prime} 53.4^{\prime \prime} \\
104\end{array}$ & 45 & E & 20 & 10 & 19.79 & 0.012 & 0.034 & 15 & AC $(0)$ \\
\hline
\end{tabular}



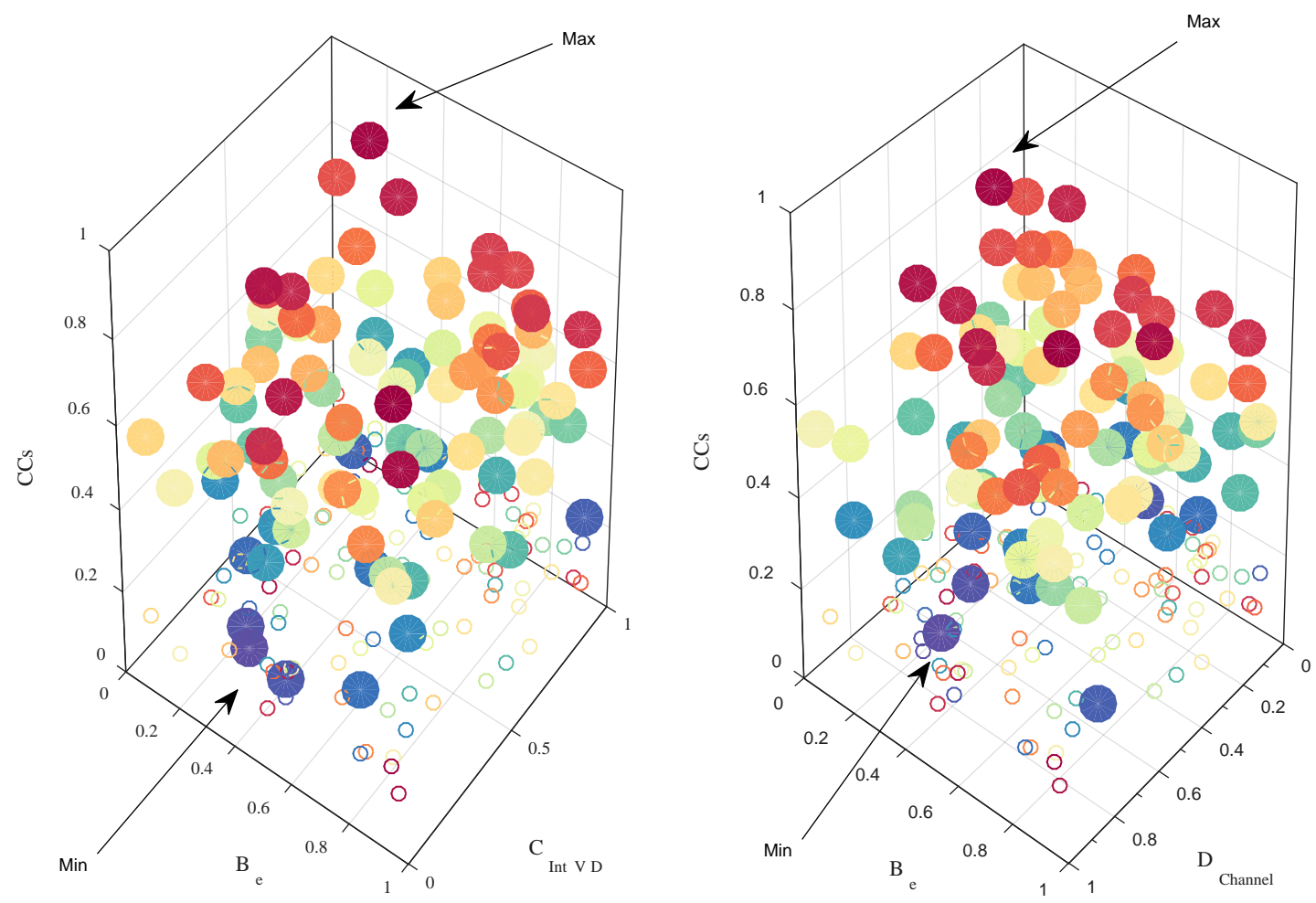

Figure 6. Comparative correlations of evaluation metrics $\left(B_{e}, \operatorname{CoV}_{C_{\text {Int }_{V_{D}}}}, D_{\text {Channel }}\right)$ on obtained $C C_{s}$ for 100 vehicles

Figure 6 illustrates the calculated values $C C_{s}$ for 100 vehicles in a comparative form to show the correlations of evaluation metrics $\left(B_{e}, \operatorname{CoV}_{C_{\text {Int } V_{D}}}, D_{\text {Channel }}\right)$ on channel status. Generally we can observe that when $\operatorname{CoV}_{C_{\text {Int }} V_{D}}$ have obtained higher values (closer to 1 , Max) and low $B_{e}$, the $C C_{s}$ of that particular vehicle obtaining higher value closer to 1 , which is the maximum. On the other hand, by looking at the right side figure in Figure 6, when both $B_{e}$ and $D_{\text {Channel }}$ getting less value closer to 0 (Min) the $C C_{s}$ getting its maximum value and vice versa. For gaining comprehend visualization, the projection of obtained $C C_{s}$ values for 100 vehicles are depicted in Figure 6.

The calculated evaluation metrics $\left(\operatorname{CoV}_{C_{I t_{V_{D}}}}, B_{e}, D_{\text {Channel }}\right.$ and $\left.C C_{S}\right)$ will be formed together in constructing the proposed evaluation matrix. Table 6 listed numerical values of these four metrics for the given example of five communicating vehicles in Figure 4. For each metric in the proposed matrix will be given an initial random weight value within the range of 0.01 (to avoid multiplication with 0 ) to 1 . The metric with positive sign will be representing the maximization while the negative sign indicating the minimization. In another words, the higher value of positive signed metric is preferable while the lower is the best in case of negative signed metrics. The below WM matrix collecting the obtained values from Table 6, the best value obtained for each computed metric is highlighted with Bold; indicating that it's the best according to that metric behaviour (Max/Min).

$$
W M\left[\begin{array}{cccc}
0.700 & 0.357 & \mathbf{0 . 1 2 0} & \mathbf{0 . 9 1 8} \\
0.370 & \mathbf{0 . 2 7 0} & 0.178 & 0.854 \\
0.550 & 1.000 & 0.890 & 0.458 \\
0.480 & 0.702 & 0.165 & 0.884 \\
\mathbf{1 . 0 0 0} & 0.366 & 0.510 & 0.625 \\
\boldsymbol{M a x}_{W 1} & \boldsymbol{M i n}_{W 2} & \boldsymbol{M i n}_{W 3} & \boldsymbol{M a x}_{W 4}
\end{array}\right]
$$


Table 6: Comparison between candidate vehicles towards evaluation metrics using proposed Weighted Matrix (WM).

\begin{tabular}{|c|c|c|c|c|c|c|c|c|}
\hline $\begin{array}{c}\text { Wieghted } \\
\text { Metrics } \\
\text { Candidate } \\
\text { Vehicles } \\
\end{array}$ & $\begin{array}{c}+0.60 \\
W_{1}\end{array}$ & & & & & $\begin{array}{c}-0.89 \\
W_{2}\end{array}$ & $\begin{array}{c}-0.49 \\
W_{3}\end{array}$ & $\begin{array}{c}+0.90 \\
W_{4}\end{array}$ \\
\hline $\begin{array}{l}\text { VEHICLE } \\
\text { ID }\end{array}$ & $\frac{C_{I n t_{V_{D}}}}{\operatorname{Max}}$ & $\begin{array}{l}\mathrm{NO} . \\
\text { recieved } \\
B / s\end{array}$ & $\begin{array}{c}\text { Percentage of } \\
\text { B-received }\end{array}$ & $\begin{array}{l}\text { Average of } \\
\text { Received } B \\
\sum^{n} \frac{B_{S}}{V_{n}}\end{array}$ & Variance_Breceived & $\begin{array}{c}\text { Error per } \\
\text { meter } \\
B_{e} / m\end{array}$ & $\begin{array}{c}\mathrm{D}_{\text {Channel }} / \mathrm{Se} \\
\text { conds }\end{array}$ & CC_S \\
\hline Source & 0.700 & 8 & $80 \%$ & 6 & 0.250 & 0.357 & 0.120 & 0.918 \\
\hline A & 0.370 & 6 & $60 \%$ & 6.6 & 0.100 & 0.270 & 0.178 & 0.854 \\
\hline B & 0.550 & 4 & $40 \%$ & 6.2 & 0.550 & 1.000 & 0.890 & 0.458 \\
\hline $\mathrm{L}$ & 0.480 & 8 & $80 \%$ & 5.3 & 0.337 & 0.702 & 0.165 & 0.884 \\
\hline Destination & 1.000 & 6 & $60 \%$ & 3.8 & 0.366 & 0.366 & 0.510 & 0.625 \\
\hline \multicolumn{6}{|c|}{$\begin{array}{l}\text { * Positive (+) Indicates the higher value is preferred } \\
\text { * Negtive (-) Indicates the lower value is preferred }\end{array}$} & $\begin{array}{l}\sum_{i=0}^{n} B_{e} \\
=1.353\end{array}$ & & \\
\hline
\end{tabular}

Table 7: WM after applying normalization process

\begin{tabular}{|c|c|c|c|c|c|c|c|c|c|}
\hline $\begin{array}{l}\text { VEHICLE } \\
\text { ID }\end{array}$ & $\begin{array}{l}C_{\text {Int }_{V_{D}}} \\
\text { Step1 }\end{array}$ & $\begin{array}{l}C_{\text {Int }_{V_{D}}} \\
\times W_{1} \\
\text { Step2 }\end{array}$ & $\begin{array}{c}B_{e} \\
\text { Step1 }\end{array}$ & $\begin{array}{c}B_{e} \times W_{2} \\
\text { Step2 }\end{array}$ & $\begin{array}{c}\mathrm{D}_{\text {Channel }} \\
\text { Step1 }\end{array}$ & $\begin{array}{l}\mathrm{D}_{\text {Channel }} \\
\times W_{3} \\
\text { Step2 }\end{array}$ & $\begin{array}{l}\text { CC_s } \\
\text { Step1 }\end{array}$ & $\begin{array}{c}C C_{-} s \times \\
W_{4} \\
\text { Step2 }\end{array}$ & $\begin{array}{l}\text { Evaluation } \\
\text { Matrix Score } \\
\text { (EMS) } \\
\text { Vehicle Rank }\end{array}$ \\
\hline Source & 0.700 & 0.420 & 0.756 & 0.672 & 1 & 0.490 & 1 & 0.900 & R1 2.482 \\
\hline A & 0.370 & 0.222 & 1 & 0.890 & 0.674 & 0.330 & 0.930 & 0.837 & R2 2.279 \\
\hline B & 0.550 & 0.330 & 0.270 & 0.240 & 0.134 & 0.065 & 0.498 & 0.449 & R5 1.084 \\
\hline $\mathrm{L}$ & 0.480 & 0.288 & 0.384 & 0.341 & 0.727 & 0.356 & 0.962 & 0.866 & R4 1.852 \\
\hline Destination & 1 & 0.600 & 0.737 & 0.655 & 0.235 & 0.115 & 0.680 & 0.612 & R3 1.983 \\
\hline $\begin{array}{c}\text { VEHICLE } \\
\text { ID }\end{array}$ & $\begin{array}{l}\text { Index } \\
\text { value of } \\
\text { Metric } \\
\text { (IVM) } \\
\text { Rank }\end{array}$ & R3 1.860 & & $\begin{array}{r}\text { R2 } \\
3.147\end{array}$ & & $\begin{array}{r}\mathrm{R} 4 \\
1.357\end{array}$ & & $\begin{array}{r}\text { R1 } \\
3.665\end{array}$ & Step3 \\
\hline
\end{tabular}

The following steps are to normalize the values of $W M$ to gain a unified behaviour form, 1 indicating the best value for both Min/Max metrics. This was processed by first, (step1), dividing the value of every evaluation metric of each vehicle by its Max highlighted value Metric $_{V_{i}} / M_{\text {ax }}$ Metric $_{i}$. For example, the $C C_{s}$ value of vehicle " $A$ " 0.854 is be divided by the Max of $C C_{s}$ metric that obtained from available vehicles in the evaluation area, which is 0.918 as listed in Table 7, step 1 $C C_{s}$. The metrics with minimization behaviour it was normalized by dividing the minimum obtained value by each value of every evaluated metric for each vehicle $\operatorname{Min}_{\text {Metric }_{i}} /$ Metric $_{V_{i}}$. For instance, the minimum value of $B_{e}$ obtained from evaluated vehicles 0.270 is divided by obtained $B_{e}$ from vehicle " $L$ " 0.702 , that is resulted with 0.384 (as listed in Table 7 , step 1). Thus, we can observe the vehicles have obtained the value 1 for any of the four evaluation metrics, which indicating that the evaluation status of that particular vehicle is good and transmission power can be adapted to get increased depending on the emergency/priority requirements. The second step 2 is to multiply each obtained value from step1 with the respected weight value of each evaluation metric in the proposed WM. The calculated values are listed in Table 7, step 2. The last step which is stpe3 is to calculate the obtained score for each vehicle by adding the calculated values from step 2 for all evaluation metrics for each vehicle. The Evaluation Matrix Score (EMS) is obtained in this step as listed in Table 7. It's clear that Source vehicle has obtained the highest score followed by A and others as marked with " $R_{i}$ " ( $i$ is the vehicle number). Accordingly, proposed $W M$ it works in dynamically evaluating the available vehicles in the zone of IoV activities. 
On the other hand, to adapt the transmission power of a vehicle after the evaluation processes have been conducted using $W M$, initially the received value of MinP from beacon it will be used together with MaxP in order to make an estimation of the optimum value of the transmission power that vehicle should use in broadcasting its beacons in time $t+1$. To achieve that we have suggested to take the average value of $\operatorname{MinP}$ and $\operatorname{MaxP}$ to be averaged with the obtained value of $T P A_{\text {Power }_{\text {val }}(t)}$ after deduction from circuit power consumption $P_{c}$ and divided by $1+\beta$, where $\beta$ equals to 0.2884 . The idea is that the vehicle should adjust its maximum power be maintained in such a way to decrease the channel congestion at the same time it should not be underutilized the transmission power, which could lead to a case where the beacons cannot arrive to further neighbors. The wise power adaption can maintain further awareness in case of emergency alerts to avoid chain of accidents phenomena, which is the main intention of having IoV technology. Equation 8 is calculating the TPA_val. It is worth mentioning that we have set an interval of 10 seconds to update the value of TPA_val after new channel evaluation is performed when the new piggybacked information has been received from neighboring vehicles.

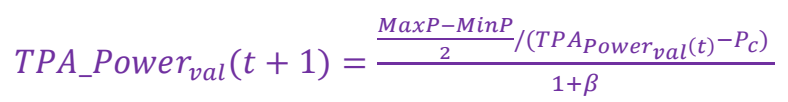

In order to present the numerical behaviour of our proposed TPA scheme, Figure 7 illustrating the calculated TPA_val for 100 vehicles. It started with high transmission power of 1.49 Watt, (around 32dBm) eventually, our proposed scheme could maintain an average power of 0.8 Watt with 100 vehicles.

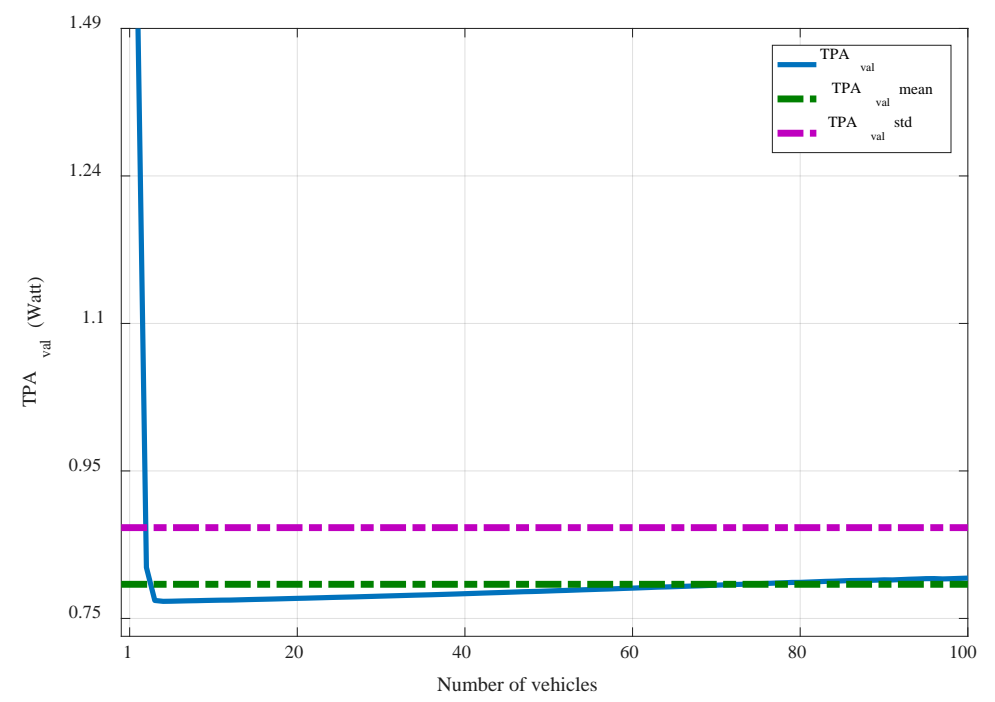

Figure 7. Calculated TPA_val for 100 vehicles

Figure 8 shows the pseudocode of proposed TPA, where it starts with constructing the proposed WM and the steps of calculating the piggybacked information that integrated into beacons. Each metric at the initial stage will obtain a random value generated within an interval between 0 to 1 . Afterwards, the IVM value will be calculated for each metric of each vehicle in the evaluation area and inserted accordingly into the $W M$ (as presented in Table 7). The EMS value will then be calculated for each vehicle using the inserted IVM value of each metric. For the reference of evaluation, the vehicle that has obtained highest score $\left(E M S_{\text {Max }}\right)$ from the evaluation using $W M$ will be ranked as first followed by the rest whose obtained lower score. 


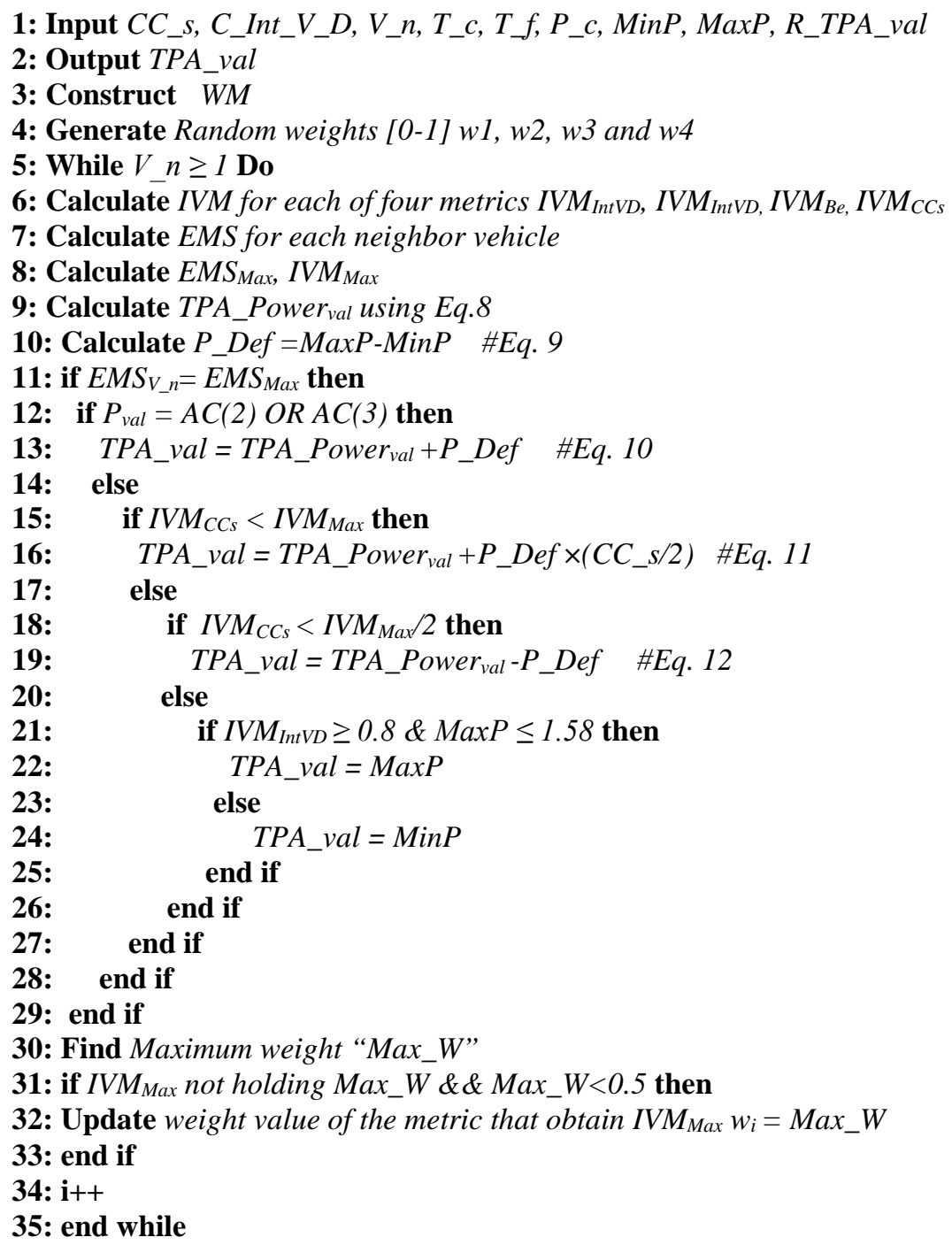

Figure 8: Pseudocode of proposed TPA

Similarly, to evaluate the impact of each metric the score will be calculated, that show its importance in indicating the rank of a vehicle considering the four metrics $\left(C \_I n t \_D, B \_e, D \_\right.$channel and $\left.C C \_s\right)$, and thus the power value of a vehicle will be adjusted accordingly. For instance, when the $I V M_{C C s}$ is calculated as the highest value, this indicating that the current channel status has more impact on deciding the upcoming power value TPA_val to be used by vehicle $i$, hence this metric will be dynamically assigned with the maximum weight value. On the other hand, when a vehicle has obtained high EMS score (which means has obtained high score in average of four evaluated metrics), TPA algorithm decides to increase that vehicle transmission power by adding the deference of power obtained by Equation 9 and calculated TPA_val after meet the condition in Line no. 12 in Figure 8.

It's important to mention that we have considered the priority of beacons by ignoring the estimated TPA_val and keep on increasing the power value when the $P_{-}$val is either $A C(3)$ or $A C(2)$ (voice or video emergency messages), refer to Tables 8 and 9. The TPA_val also will be decreased after analyzing the channel status of each vehicle and the evaluated $I V M_{C C S}$ score is bellow than maximum, to ensure that new estimated TPA_val can maintain the power using Equation 10 without creating further increment that leads in degrading the channel status.

On the other hand, the other considered scenario by our proposed TPA scheme is that when the CC_s is obtaining an optimum value equals to one. This indicating that the wireless channel is without any congestion reported after the analysis 
was conducted using our proposed WM. At this scenario, the TPA scheme will consider the $C_{-}$Int- $V_{-} D$, that collected from piggybacked information, when the $C_{-}$Int $-V_{-} D$ is more than 0.8 , TPA scheme allows the vehicle to apply maximum power by substituting the value of MaxP into TPA_val. Logically this value would not cause any possible congestion when the crowding distances between vehicles are not very close, otherwise, our proposed TPA scheme is assuming the TPA_val is $1.58 \mathrm{~W} \approx 32 \mathrm{dBm}$ as a maximum transmission power suggested by [15]. Otherwise, when the $C_{-}$Int- $V \_D$ in the range bellow 0.8 and MaxP less than 32dBm, this indicates that there will be vehicles positioned in closer distance (high dense environment), could use transmission power less than required to reach other vehicles, thus the TPA_val will be counted as follows:

$$
\begin{aligned}
& P_{D e f}=\operatorname{Max} P-\operatorname{MinP} \\
& \left(\text { Case-1) TPA } A_{\text {val }}=T P A_{-} \text {power } r_{v a l}+P_{D e f}\right.
\end{aligned}
$$

After calculating the transmission power using Equation 8, the power adjustment will be applied in three different cases as formed by Equations 10-12. By referring to proposed algorithm of TPA scheme in Figure 8, the power will be slightly increasing with the small value of $P_{D e f}$ when the two conditions are met $\left(E M S_{V \_n}\right.$ is the highest value and vehicle $i$ has to send a high priority message). While in the second case, the obtained power will be fractionally decreased when evaluation metric CCs in WM is not behaving the best compare with other three metrics. Third case the power will keep on linearly decreasing with the value of $P_{D e f}$ when the channel status CCs dropping bellow 0.5, which indicates quality below average thus power should be decreasing to mitigate any possible congestion.

$$
\begin{aligned}
& \left(\text { Case-2) } T P A_{\text {val }}=T P A_{\text {power }_{\text {val }}}+P_{\text {Def }} \times \frac{C C_{S}}{2}\right. \\
& \left(\text { Case-3) } T P A_{\text {val }}=T P A_{\text {power }_{\text {val }}}-P_{\text {Def }}\right.
\end{aligned}
$$

Table 8. Message Priority Value $\left(\mathrm{P}_{\mathrm{val}}\right)$ [28].

\begin{tabular}{|c|c|}
\hline Priority (traffic in EDCA) & Message Type for VANET \\
\hline $\begin{array}{c}\text { Priority 1 (Voice traffic - } \\
\text { AC(3)) }\end{array}$ & $\begin{array}{c}\text { Accident message, messages } \\
\text { from emergency vehicle }\end{array}$ \\
\hline $\begin{array}{c}\text { Priority 2 (Video traffic - } \\
\text { AC(2)) }\end{array}$ & $\begin{array}{c}\text { Accident indication } \\
\text { messages, sensing vehicle } \\
\text { tyres }\end{array}$ \\
\hline $\begin{array}{c}\text { Priority3 (Best-effort traffic } \\
\text {-AC(1)) }\end{array}$ & $\begin{array}{c}\text { Warning related messages, } \\
\text { e.g. work ahead, school area } \\
\text { ahead, etc. }\end{array}$ \\
$\begin{array}{c}\text { Priority } 4 \text { (Background traffic } \\
\text { - AC }(0) \text { ) }\end{array}$ & $\begin{array}{c}\text { General messages e.g. } \\
\text { periodic broadcast messages }\end{array}$ \\
\hline
\end{tabular}

Table 9. PRIORITY SPECIFIC PARAMETERS [29].

\begin{tabular}{|c|c|c|c|}
\hline AC & CWmin & CWmax & AIFS \\
\hline 0 & CWmin & CWmax & 2 \\
\hline 1 & CWmin & CWmax & 1 \\
\hline 2 & $($ CWmin+1)/2 & CWmin & 1 \\
\hline 3 & $($ CWmin+1)/4- & $($ CWmin+1)/2 & 1 \\
& 1 & & \\
\hline
\end{tabular}

\subsection{SIMULATION AND PERFORMANCE ANALYSIS}

In this section, the simulation settings and performance analysis are presented and discussed. The simulation is conducted using OMNeT++ [30] joined with MiXiM framework v2.3 [31]. In order to simulate the behavior of 802.11p protocol, we have hardly tried to follow the exact parameters of IEEE 80.11 MAC module that was specified by 802.11p [31]. We have also included into our simulated 802.11 MiXiM physical layer the Bit Error Rates (BER) and Packet Error Rates (PER), that were considered during all transmission bit rates which are implemented in our experiments. It's important mentioning that we have set the center frequency to $5.9 \mathrm{MHz}$ and Access Category (AC) was equal to zero. Figure 9 presents the simulate road map that was integrated to OMNEeT++ simulator using Simulation of Urban MObility (SUMO). As a way 
to model the utilized signal propagation, a log-normal shadowing model [32] was employed. An exponent path loss of 3.5 with standard deviation 6 is applied to our simulated scenario.

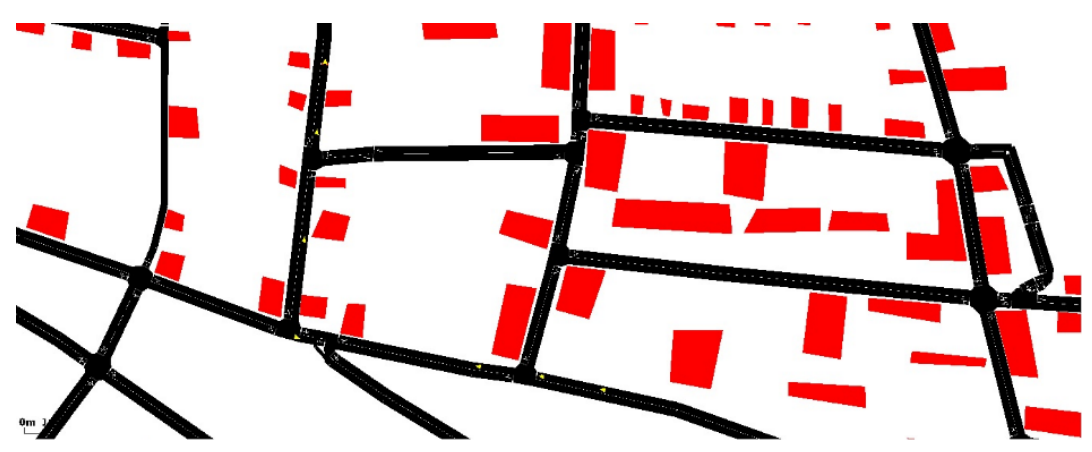

Figure 9. Simulated road map.

We have compared the overall throughput, the average congestion rate with respect to vehicle density and the probability of received beacons per $T$ for all messages and for the highest priority message for 150 vehicles. A packet size of 512 Bytes and rate of 10 packets per second were set for the generated traffic during the simulation time. It's also worth mentioning that when a case of priority message, the QoS parameters, and corresponding default values will be implemented as listed in Tables 8 and 9. We have simulated our proposed TPA scheme as well as other most related work, which is a joint adaptation scheme proposed by Danda [15], that we represented as (default method), for the duration of 35 minutes. Moreover, it's important to shed the light on that all collected results are the average of 5 simulation runs.

Figure 10 illustrates the overall throughput that was achieved during simulation time. It's obvious that our proposed TPA scheme could achieve higher overall throughput during the simulation time compare with the default method. At the early time of simulation, the overall throughput was the same for both schemes. This was due to the reason that at the initial stage of simulation most of the vehicles are not able to identify their neighbors since the vehicle density was very low at that stage. Thus, our proposed scheme at that time could not adapt its transmission power. Afterwards, we can observe that our proposed TPA scheme started to outperform the default method after 14 minutes of simulation time towards the end of the simulation. The reason was that our proposed TPA scheme has decreasing the transmission power whenever it sensed that vehicle density is getting higher. In other words, when the number of vehicles increases (represented with $C_{-}$Int $-V_{-} D$ ) the probability of congestion is increased similarly, thus the TPA scheme has decreased the transmission power in a way to allow the high priority messages to get transmitted successfully.

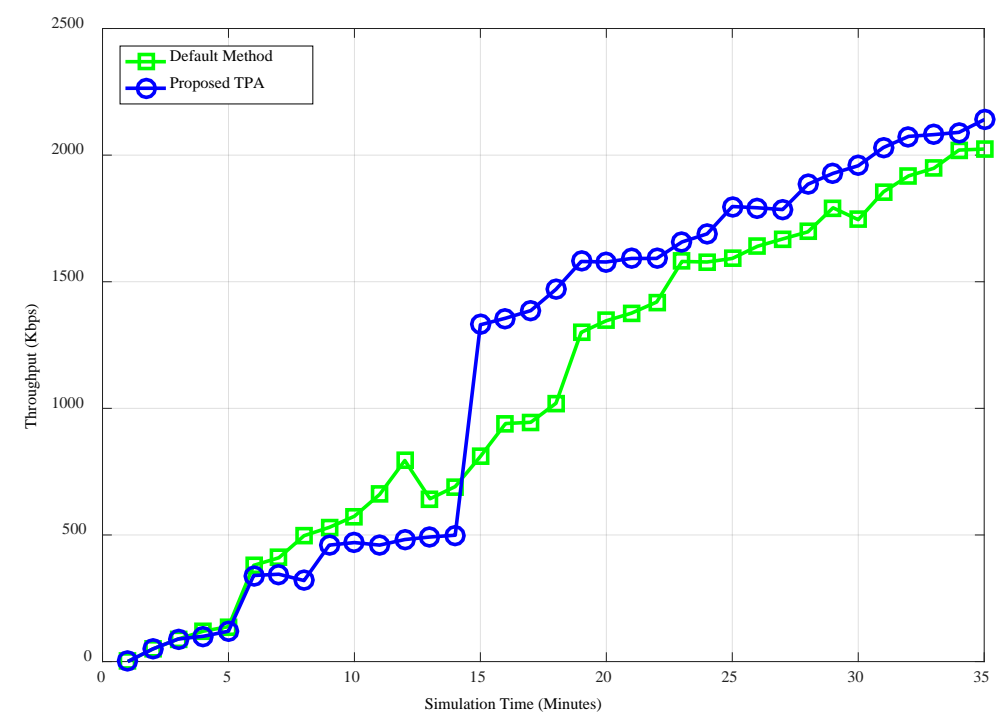

Figure 10. Overall throughput during simulation time. 
On the other hand, TPA increased the transmission power when the number of vehicles is decreased. Throughout this mechanism, top priority messages have obtained greater opportunity to be transmitted and delivered to their destinations. To this end, TPA scheme could lower the overall network overhead since it efficiently decreased the broadcasted and rebroadcasted messages within each road section. Hence, we can state that although TPA scheme adopting a modified beacon frame with piggybacked information the overall achieved throughput using our proposed scheme is improved compared to the default method.

Figure 11 shows the average congestion rate of our proposed TPA scheme against the default method. Our TPA shows the lowest congestion rate compared with the default method and is the steadier as this congestion rate remained in average below 0.1 during the entire simulation time. On the other hand, the default method has achieved not as good as results since it kept increasing the congestion rate by the time vehicle density is increasing (vehicle/km). We can relate that to the aggressive change in $T x$ parameters when switching between the different channel states. We refer the reader to [33] for more details about $D C C$ schemes.

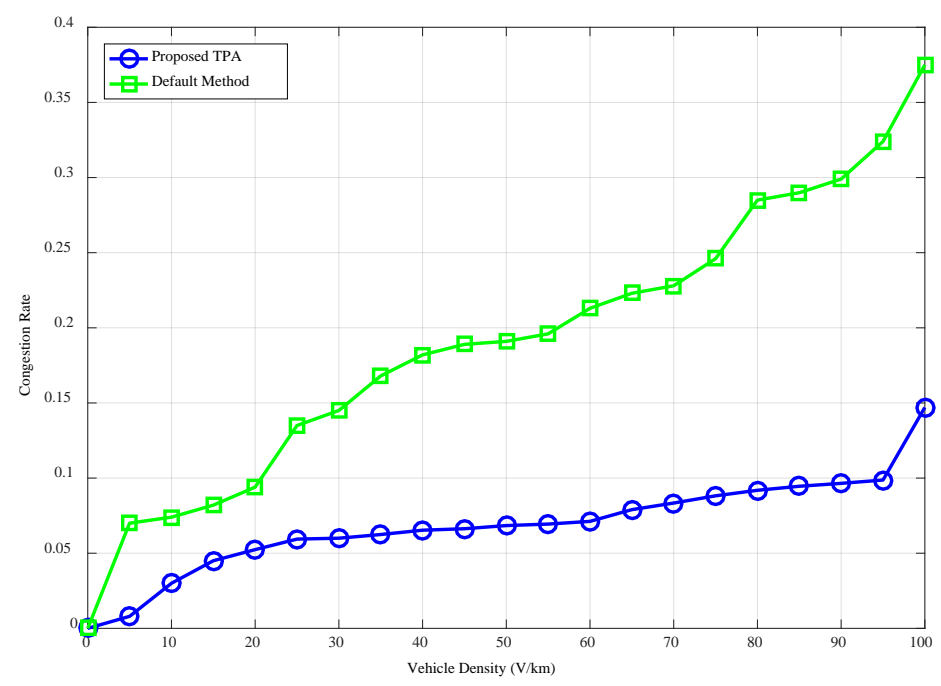

Figure 11. Average congestion rate vs. V/Km.

It is essential here to highlight the significance of a low congestion rate to attain a better intensity of awareness in vehicular surroundings. In other words, our proposed TPA scheme could achieve low average congestion rate by maintaining an adaptive transmission power, so that when the power is decreased for a short period does not mean it reduced the level of awareness, since more beacons can be lost whenever the congestion occurs. Therefore, we can interpret from results presented in Figure 11 that our TPA scheme can ensure the safety and top priority messages to be arrived at their destination efficiently.

In order to analyze the probability of beacons which have reached their destination successfully $\left(\mathrm{P}_{\mathrm{br}(T)}\right)$, Figure 12 demonstrating the beacon recipient rate in a probabilistic form. We measured the probability of beacon recipient rate, which is expressing the fraction of beacons that have reached the destination at $T$ seconds, using our proposed TPA scheme and default method. It's obvious that both methods have an inverse exponential behavior in the average collected $\mathrm{P}_{\mathrm{br}(T)}$ that are decreasing as a function of distance, although our TPA scheme could maintain better average $\mathrm{P}_{\mathrm{br}(T)}$ compare with default scheme. 


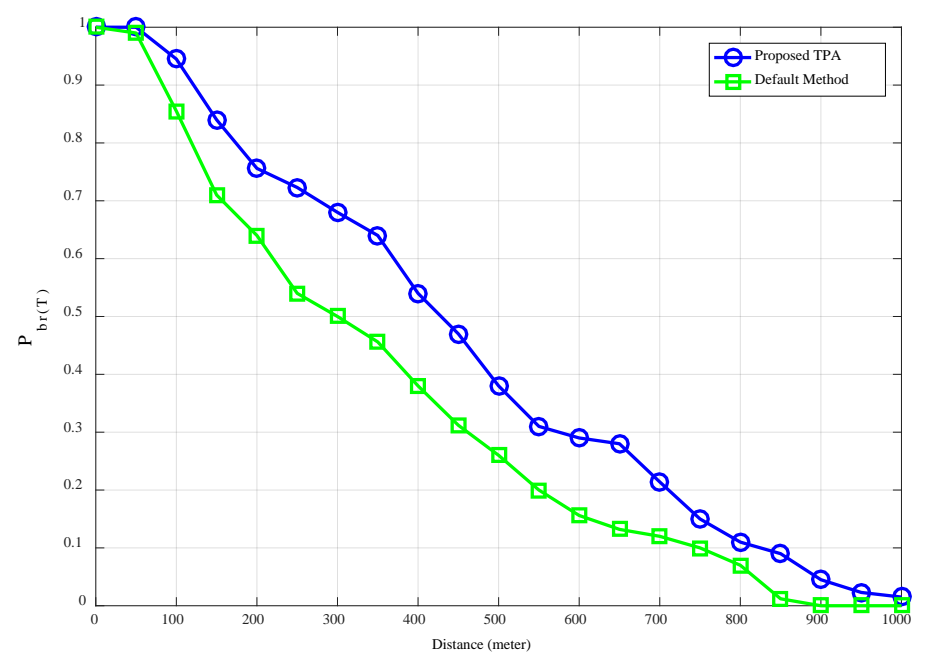

Figure 12 . The probability of beacon recipient rate vs. $C \_I n t-V \_d$.

It's critical mentioning that the presented $x$-axis in Figure 12 representing the $C \_I n t-V \_d$ is per meter. The inter-vehicle distance was exponentially distributed, that indicates the given values are representing the mean of sets from $10,20 \ldots 1000$ using Cumulative Distribution Function (CDF). Thus, whenever inter-vehicle distance is exponentially increasing this will decrease $\mathrm{P}_{\mathrm{br}(T)}$ due to possible loss that would happen within wireless inter-vehicle communication. This resulted in more dropped beacons and then after degrades the vehicular awareness in VANET environment, which is the main objective need be achieved. We can justify the main reason of improving $\mathrm{P}_{\mathrm{br}(T)}$ using our TPA scheme is that the adaptive transmission power concept with the respect of given priority for message type, channel statues, and crowding inter vehicle distance have contributed more in increasing the utilized power in order to enrich the beacons among vehicles. Its fact that when the crowding inter-vehicle distance is getting higher this infers that vehicle density within a given road segment is low, thus increasing the transmission power would not result in possible channel congestion. Due to these reasons, our proposed TPA scheme could improve in average the performance by achieving higher $\mathrm{P}_{\mathrm{br}(T)}$ compare to default method.

In order to reflect the impact of $\mathrm{D}_{\text {Channel }}$ evaluation metric, Figure 13 demonstrates the probability of channel-busy $\mathrm{P}_{(\text {Channel- }}$ Busy) against the vehicle density (V/km). We have compared our proposed TPA scheme along with default method in order to present the improvement that has achieved. We can observe that the $\mathrm{P}_{\text {(Channel-Busy) }}$ of the default method was increasing with vehicle density was getting higher. The control channel utilization with default method at $30 \mathrm{~V} / \mathrm{km}$ shown as underutilization while with higher vehicle densities, $150 \mathrm{~V} / \mathrm{km}$ it was over utilized.

In contrast, our TPA scheme initially estimated the beacon generation rate with respect to priority messages afterward raised the transmission power in order to maximize the vehicular awareness. Thus, in average it's clear that our TPA scheme could efficiently utilize the control channel under acceptable level with the increasing of vehicle density up to maximum level, $150 \mathrm{~V} / \mathrm{km}$. 


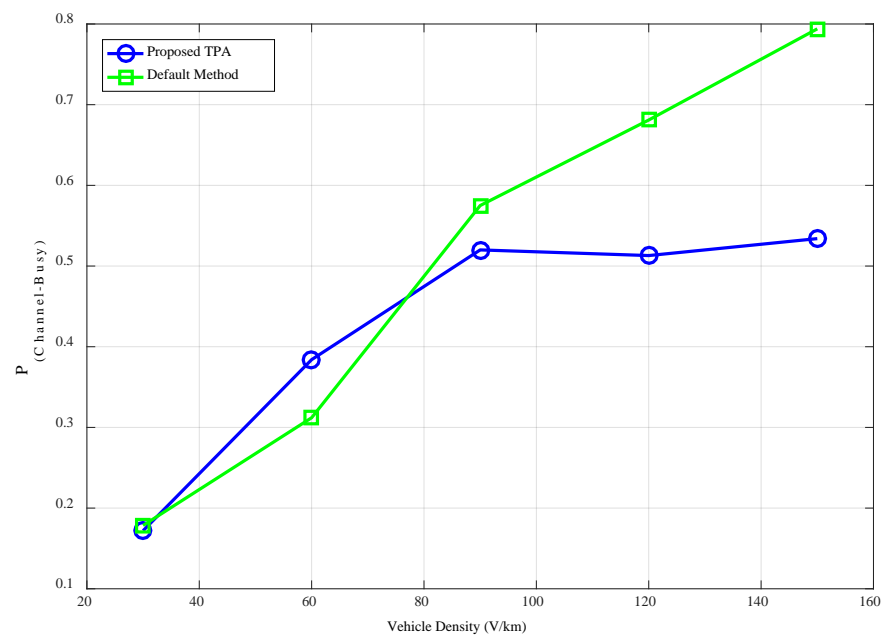

Figure 13. Probability of channel-busy vs. V/Km

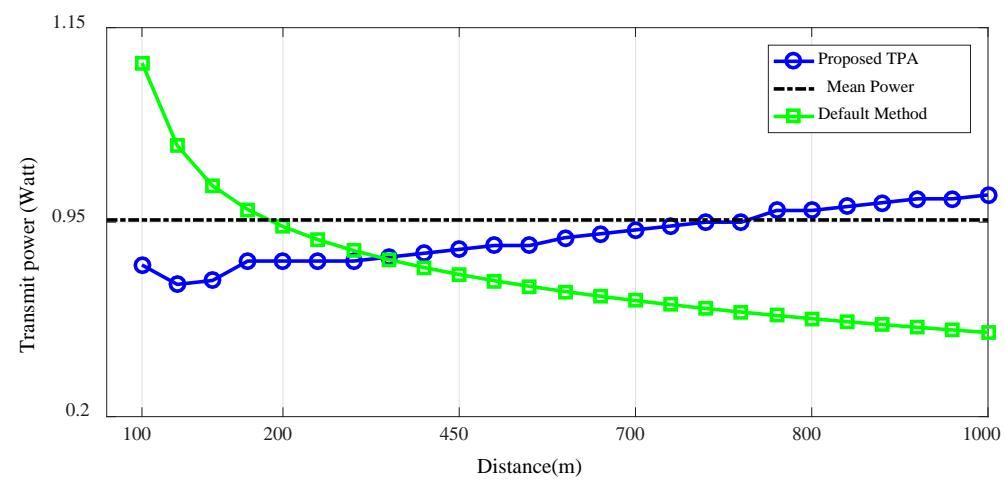

Figure 14. Average transmit power vs. distance

On the other hand, to evaluate the proposed TPA scheme in maintaining transmission power, the average power in Watt was collected during simulation in comparison with existing method and depicted in Figure 14. The power utilized by vehicles in transmitting messages was measured with respect to distance variance. As a general reading from Figure 14 we can interpret that when the $C \_I n t-V \_d$ is low (100 to 500 meter) TPA scheme set the transmit power to an average below 0.9 Watt $\approx 29.6 \mathrm{dBm}$, while the existing method started with an average high power and turned to decreasing it by the time distance is increasing. In contrast, our proposed scheme could maintain the transmit power with a mean power of 0.95 Watt $\approx 29.8 \mathrm{dBm}$ and slightly increased after 800 meters $C_{-}$Int $-V_{-} d$. Hence, this result proved that our proposed scheme behaved more wisely in saving in average the transmit power with changing of mobility factor represented by $C \_I n t-V \_d$. This is due to the fact that when IVM rank of $C_{-}$Int $-V_{-} d$ evaluation metric in the proposed WM getting higher score, leading to indicate that this metric is highly dynamic and the power value should be adjusted accordingly. 


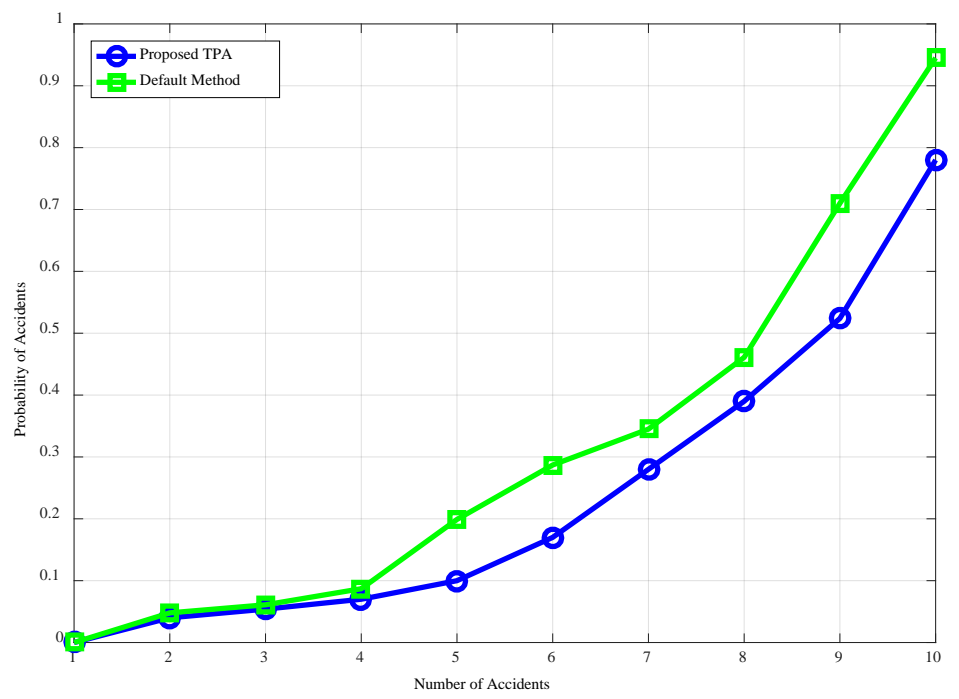

Figure 15. Probability of accidents vs. Number of accidents

Finally, in order to show the ability of our proposed TPA scheme in reducing the number of accidents compares with the state of the arts, a probabilistic model using Equation 13 is conducted. We have modeled the number of vehicles in a tested sequence of accidents as 10 which are exponentially distributed along the sequence. The $\lambda$ parameter of the exponential distribution was designated from the value between 10 and $100 \mathrm{~V} / \mathrm{Km}$. On the other hand, the velocity of vehicles chose to be in a normal distribution with a mean of $100 \mathrm{Km} / \mathrm{h}$ and standard deviation $20 \mathrm{Km} / \mathrm{h}$. Whereby, every vehicle's length is also was chosen to be as four meters and its deceleration is selected as uniformly from the range 3.8 to $8.2 \mathrm{~m} / \mathrm{s}$. Thus, based on Equation 10, where $v 1$ infers the first vehicle of the sequence's accident and $N$ denotes to the sequence's accident size. As illustrated in Equation 13, in order to calculate the accident probability of $a c$ accidents in a sequence, hence we should have a probability of $a c-1$ accidents in a sequence first.

$$
P_{a}(v 1, a c, N)=\left\{\sum_{i=v 1}^{N-n c}\left[P_{v i} \times\left(\prod_{j=v 0}^{i-1}\left(0+P_{v j}\right)\right) \times P(i+1, a c-1, N)\right], a c=0\right.
$$

Therefore, out of Figure 15, we can obviously read the improvements that were achieved by our proposed TPA scheme compared with the state of the arts. Generally speaking, our proposed TPA could efficiently maintain the lower probability of accidents compared with default method. The experiment was conducted while accidents are set to be initiated during the running time with an exponential increasing up to 10 incidents within a sequence's accident. The main objective of this evaluation is to testify the ability of the proposed scheme in avoiding the chain of accidents when a vehicle crash has taken place and the safety messages triggered to disseminate the safety warning. We can refer this improvement due to the fact that our proposed scheme could significantly achieve an adaptive power during the occurrence of accidents via an accurate evaluation of channel and mobility metrics using proposed $W M$, which could assist in obtaining the optimal power that can ensure safety messages reachability with less congestion rate.

\subsection{CONCLUSION}

Approaching the appropriate transmission power is quite significant in IoV, the proposed TPA scheme could maintain efficiently the transmission power which decreased the channel congestion and thus leveraged the safety application's performance by accurately considering weighted matrix in evaluating vehicle's wireless channel characteristic and GEO information that is transmitted over piggybacked beacons. Every 10 second of evaluation interval, every vehicle analysed the received information and calculated the new adjusted transmit power value using our TPA scheme. We have introduced 
a modified beacon frame to piggybacked the analysed information that consists of required values that used in predict proactively the upcoming channel status. That was obtained by considering evaluation metrics which are the beacon transmission error rate, crowding inter-vehicle distance, in addition to beacon forwarding and carrying time delays (channel delay). The obtained predicted channel status and GEO information are used to adjust the transmission power for the top priority safety messages to reach an optimal power value without decreasing their performance in disseminating safety messages for IoV awareness. The simulation results show that our proposed TPA scheme perform better compared with an existing method in terms of overall throughput, average beacon congestion rate, the probability of beacon recipient rate and the probability of channel-busy in addition to decreasing the probability of accidents. Our future work will be more focusing on developing more specific mathematical modeling of our proposed TPA scheme with the effects of channel randomness behaviour.

\section{REFERENCES}

[1] C. Chen, C. Dong, F. Wu, S. Tang, Y. Qu, and H. Wang, "The Power of Flexible SlidingWindow: Adaptive Network Coding inWireless Mesh Networks," Adhoc \& Sensor Wireless Networks, vol. 29, 2015.

[2] H.-W. Kim and A. Kachroo, "Low Power Routing and Channel Allocation of Wireless Video Sensor Networks Using Wireless Link Utilization," Adhoc \& Sensor Wireless Networks, vol. 30, 2016.

[3] A. S. Sadiq, T. Z. Almohammad, R. A. B. M. Khadri, A. A. Ahmed, and J. Lloret, "An Energy-Efficient CrossLayer approach for cloud wireless green communications," in Fog and Mobile Edge Computing (FMEC), 2017 Second International Conference on, 2017, pp. 230-234.

[4] M. K. Khan, M. Shiraz, K. Zrar Ghafoor, S. Khan, A. Safaa Sadiq, and G. Ahmed, "EE-MRP: Energy-Efficient Multistage Routing Protocol for Wireless Sensor Networks," Wireless Communications and Mobile Computing, vol. 2018, 2018.

[5] A. S. Sadiq, K. A. Bakar, K. Z. Ghafoor, J. Lloret, and R. Khokhar, "An intelligent vertical handover scheme for audio and video streaming in heterogeneous vehicular networks," Mobile Networks and Applications, vol. 18, pp. 879-895, 2013.

[6] M. Torrent-Moreno, J. Mittag, P. Santi, and H. Hartenstein, "Vehicle-to-vehicle communication: fair transmit power control for safety-critical information," IEEE Transactions on Vehicular Technology, vol. 58, pp. 36843703, 2009.

[7] K. Z. Ghafoor, M. A. Mohammed, J. Lloret, K. A. Bakar, and Z. M. Zainuddin, "Routing protocols in vehicular ad hoc networks: survey and research challenges," Network Protocols and Algorithms, vol. 5, pp. 39-83, 2013.

[8] K. Z. Ghafoor, J. Lloret, K. A. Bakar, A. S. Sadiq, and S. A. B. Mussa, "Beaconing approaches in vehicular ad hoc networks: a survey," Wireless personal communications, vol. 73, pp. 885-912, 2013.

[9] K. Z. Ghafoor, J. Lloret, A. S. Sadiq, and M. A. Mohammed, "Improved geographical routing in vehicular ad hoc networks," Wireless Personal Communications, vol. 80, pp. 785-804, 2015.

[10] K. A. Bakar, K. Z. Ghafoor, M. A. Mohammed, A. S. Sadiq, and J. Lloret, "Vehicular cloud computing: trends and challenges," Mobile Networks and Cloud Computing Convergence for Progressive Services and Applications, vol. 262, 2013.

[11] G. Samara, S. Ramadas, and W. A. Al-Salihy, "Safety message power transmission control for vehicular ad hoc networks," arXiv preprint arXiv:1007.3058, 2010.

[12] P.-C. Cheng, K. C. Lee, M. Gerla, and J. Härri, "GeoDTN+Nav: Geographic DTN Routing with Navigator Prediction for Urban Vehicular Environments," Mobile Networks and Applications, vol. 15, pp. 61-82, 2010.

[13] M. Torrent-Moreno, "Inter-vehicle communications: assessing information dissemination under safety constraints," in Wireless on Demand Network Systems and Services, 2007. WONS'07. Fourth Annual Conference on, 2007, pp. 59-64.

[14] J. Mittag, F. Thomas, J. Härri, and H. Hartenstein, "A comparison of single-and multi-hop beaconing in VANETs," in Proceedings of the sixth ACM international workshop on VehiculAr InterNETworking, 2009, pp. 69-78.

[15] D. B. Rawat, D. C. Popescu, G. Yan, and S. Olariu, "Enhancing VANET performance by joint adaptation of transmission power and contention window size," IEEE Transactions on Parallel and Distributed Systems, vol. 22, pp. 1528-1535, 2011.

[16] L. Bao and S.-H. Yang, "New Channel Access Coordination Functions in Large Scale Wireless LAN Systems," Network Protocols and Algorithms, vol. 1, pp. 16-40, 2009. 
[17] T. Zhang, H. Antunes, and S. Aggarwal, "Defending connected vehicles against malware: Challenges and a solution framework," IEEE Internet of Things Journal, vol. 1, pp. 10-21, 2014.

[18] Y. R. B. Al-Mayouf, N. F. Abdullah, M. Ismail, S. M. Al-Qaraawi, O. A. Mahdi, and S. Khan, "Evaluation of efficient vehicular ad hoc networks based on a maximum distance routing algorithm," EURASIP Journal on Wireless Communications and Networking, vol. 2016, p. 265, 2016.

[19] S. Narayanaswamy, V. Kawadia, R. S. Sreenivas, and P. Kumar, "Power control in ad-hoc networks: Theory, architecture, algorithm and implementation of the COMPOW protocol," in European wireless conference, 2002, pp. 156-162.

[20] X. Guan, R. Sengupta, H. Krishnan, and F. Bai, "A feedback-based power control algorithm design for VANET," in 2007 Mobile Networking for Vehicular Environments, 2007, pp. 67-72.

[21] Y. R. B. Al-Mayouf, M. Ismail, N. F. Abdullah, A. W. A. Wahab, O. A. Mahdi, S. Khan, et al., "Efficient and stable routing algorithm based on user mobility and node density in urban vehicular network," PloS one, vol. 11, p. e0165966, 2016.

[22] S. A. A. Shah, E. Ahmed, F. Xia, A. Karim, M. Shiraz, and R. M. Noor, "Adaptive beaconing approaches for vehicular ad hoc networks: a survey," IEEE Systems Journal, 2016.

[23] M. Artimy, "Local density estimation and dynamic transmission-range assignment in vehicular ad hoc networks," IEEE Transactions on Intelligent Transportation Systems, vol. 8, pp. 400-412, 2007.

[24] M. Torrent-Moreno, P. Santi, and H. Hartenstein, "Distributed fair transmit power adjustment for vehicular ad hoc networks," in Sensor and Ad Hoc Communications and Networks, 2006. SECON'06. 2006 3rd Annual IEEE Communications Society on, 2006, pp. 479-488.

[25] N. Lu, N. Cheng, N. Zhang, X. Shen, and J. W. Mark, "Connected vehicles: Solutions and challenges," IEEE internet of things journal, vol. 1, pp. 289-299, 2014.

[26] I. W. Group, "IEEE standard for information technology-Telecommunications and information exchange between systems-Local and metropolitan area networks-Specific requirements-Part 11: Wireless LAN Medium Access Control (MAC) and Physical Layer (PHY) specifications Amendment 6: Wireless Access in Vehicular Environments," IEEE Std, vol. 802, 2010.

[27] S. Yousefi, E. Altman, R. El-Azouzi, and M. Fathy, "Analytical model for connectivity in vehicular ad hoc networks," IEEE Transactions on Vehicular Technology, vol. 57, pp. 3341-3356, 2008.

[28] C. Suthaputchakun and A. Ganz, "Priority based inter-vehicle communication in vehicular ad-hoc networks using IEEE 802.11 e," in Vehicular Technology Conference, 2007. VTC2007-Spring. IEEE 65th, 2007, pp. 2595-2599.

[29] S. Committee, "Wireless LAN medium access control (MAC) and physical layer (PHY) specifications: Amendment 8: Medium Access Control (MAC) Quality of Service enhancements," IEEE Computer Society, 2005.

[30] Omnet++, "Omnet++ network simulation framework," 2017.

[31] Mixim, "Mixim," 2017.

[32] T. S. Rappaport, Wireless communications: principles and practice vol. 2: prentice hall PTR New Jersey, 1996.

[33] F. J. Ros, P. M. Ruiz, and I. Stojmenovic, "Acknowledgment-based broadcast protocol for reliable and efficient data dissemination in vehicular ad hoc networks," IEEE Transactions on Mobile Computing, vol. 11, pp. 33-46, 2012. 